\title{
Vividly-colored silicon metasurface based on collective electric and magnetic resonances
}

\author{
Wuzhou Song ${ }^{1}$, Shiqiang $\mathrm{Li}^{2}$, and Kenneth B. Crozier*1, 2 \\ 1 School of Physics, University of Melbourne, VIC 3010, Australia \\ ${ }^{2}$ Department of Electrical and Electronic Engineering, University of Melbourne, VIC 3010, Australia \\ * - Email: kenneth.crozier@unimelb.edu.au
}

\begin{abstract}
We fabricate a silicon nanorod-based metasurface that shows vivid colors. Each nanorod supports electric and magnetic dipole modes whose coupling leads to collective resonances. The reflected field is described by a classical coupled dipole model.
\end{abstract}

\section{INTRODUCTION}

There is currently much interest concerning opticallyresonant nanoparticles, mainly owing to their ability to generate substantial near-field enhancements and to modify the amplitude and phase of far-fields. Recently, experimental demonstrations have been made of resonances of all-dielectric nanoparticles of high refractive index $[1,2]$. Here, for the first time to the best of our knowledge, we demonstrate silicon metasurfaces that generate vividly-colored reflections. We show that these originate from electric and magnetic dipole modes that couple to form collective resonances.

\section{EXPERIMENT AND RESULTS}

The fabrication process begins with thinning the device layer of a silicon-on-insulator (SOI) wafer down to a thickness of $190 \mathrm{~nm}$. Nanorods are then formed by ebeam lithography and dry etching in square lattices (300 nm period, Fig.1). The substrate $\mathrm{Si}$ of the SOI wafer is then removed to prevent the reflection that would otherwise occur.

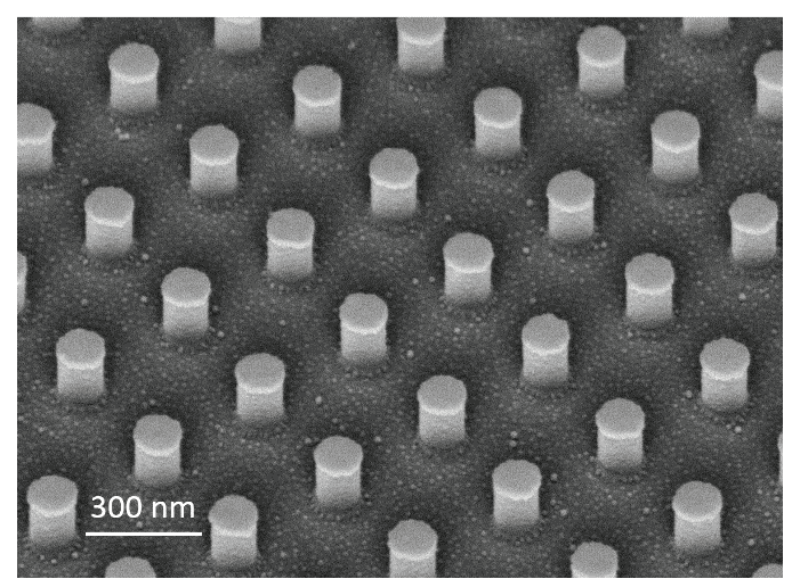

Fig. 1. SEM of a-Si nanorod array on $\mathrm{SiO}_{2}$ substrate.

Bright-field microscopy (Fig.2) reveals that the nanorod array exhibit vivid colors. With increasing nanorod diameter $(\sim 68 \mathrm{~nm}$ to $\sim 163 \mathrm{~nm})$, the color of the silicon metasurface changes from blue to red.

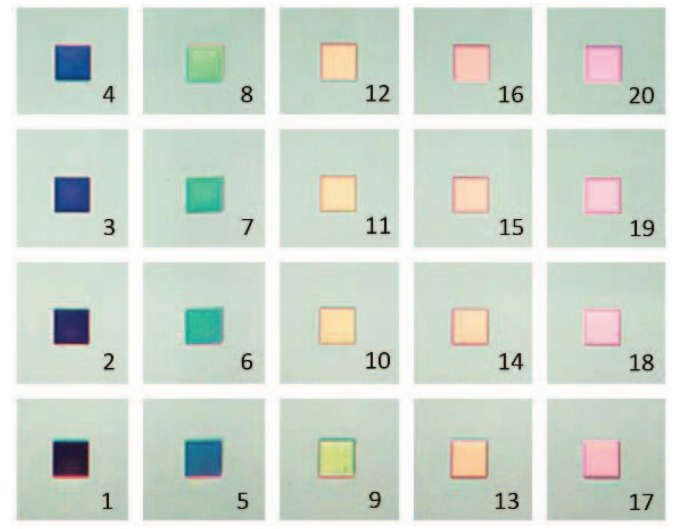

Fig. 2. Bright filed microscopy images of silicon metasurface of which where the color varies with increasing diameters.

We measure the reflectance spectra of nanorod arrays (Fig. 3), with a multilayered dielectric mirror with known reflectance used as the reference. These spectra thus represent absolute reflectance. Large reflectance is found from the nanorod arrays, particularly for the red-colored array, whose reflectance reaches $\sim 87 \%$ near $\lambda=680 \mathrm{~nm}$.

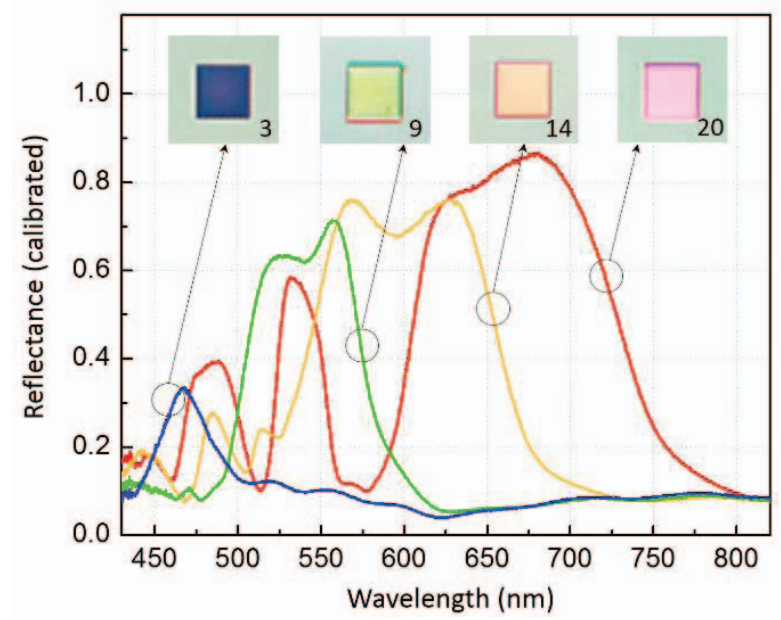

Fig. 3. Measured reflectance spectra of metasurfaces, with bright-field microscope images also shown. These generate vivid colors across the visible.

Interesting, our experiments (Fig. 4) reveal that an array of nanorods in a square lattice (300 $\mathrm{nm}$ period) produces a reflection spectrum very similar to that from nanorods in a triangular lattice (322 nm period). These arrays have the same nanorod surface density. This is different from conventional gratings for which reflectance is highly dependent on periodicity. 


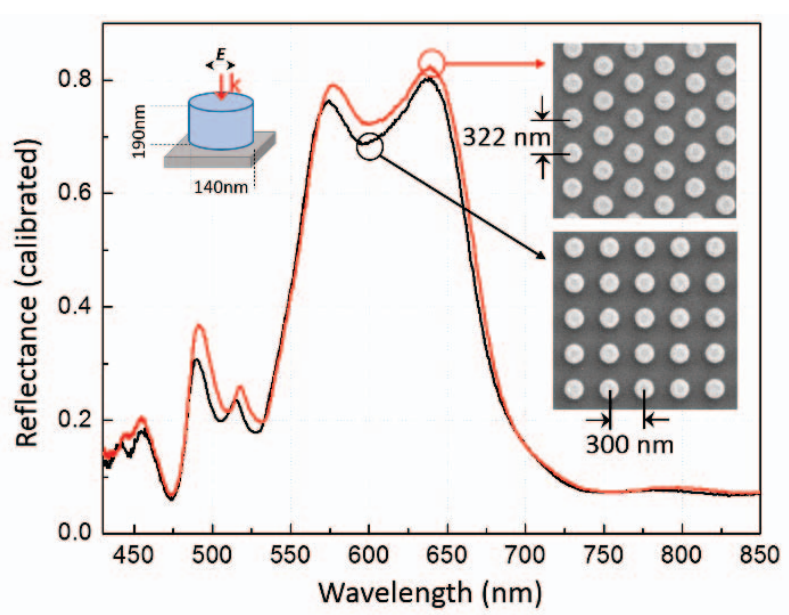

Fig. 4. Measured reflectance spectra of two different metasurfaces.

\section{Simulation AND CALCUlations}

We next consider the nature of the modes. Finitedifference time-domain simulations of the scattering cross section spectrum of a single nanorod on an $\mathrm{SiO}_{2}$ substrate show two dominant peaks (Fig.5). Examination of the field distributions (Fig.5 inset) reveals that these correspond to electric and magnetic resonances. The magnetic resonance exhibits an anti-parallel orientation of the electric field at opposite sides of the particle and a maximum of the magnetic field at its center [3].

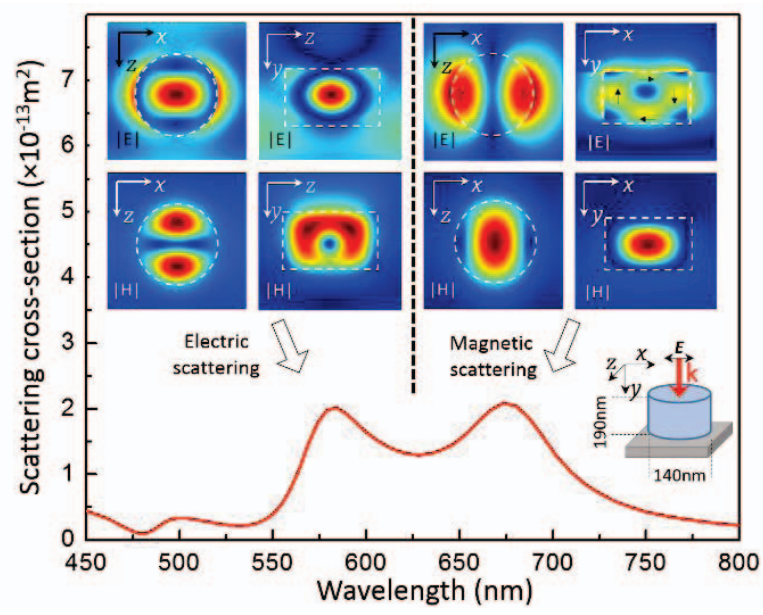

Fig. 5. Simulated scattering cross-section of a-Si nanorod from array. Insets: electric \& magnetic field profiles for electric $(\lambda=613 \mathrm{~nm}) \&$ magnetic $(\lambda=716 \mathrm{~nm})$ resonances.

We further study the nature of the modes by breaking the extinction cross section of a single $\mathrm{Si}$ nanorod into electric and magnetic dipole components. This is done by performing discrete dipole approximation (DDA) calculations, then finding the electric $\boldsymbol{P}$ and magnetic $\boldsymbol{M}$ dipole moments from the discrete dipoles $\boldsymbol{p}_{j}^{E}$ as follows:

$$
\begin{aligned}
& \mathbf{P}=\sum_{j} \mathbf{p}_{j}^{E} \\
& \mathbf{M}=\sum_{j} \frac{\omega}{2 i}\left[\left(\mathbf{r}_{j}-\mathbf{r}_{0}\right) \times \mathbf{p}_{j}^{E}\right]
\end{aligned}
$$

where $\boldsymbol{r}_{j}$ is the position vector of the $j$ th dipole, and $\boldsymbol{r}_{0}$ is the position vector for disk center. Fig. 6 confirms the electric and magnetic dipole nature of the extinction peaks.

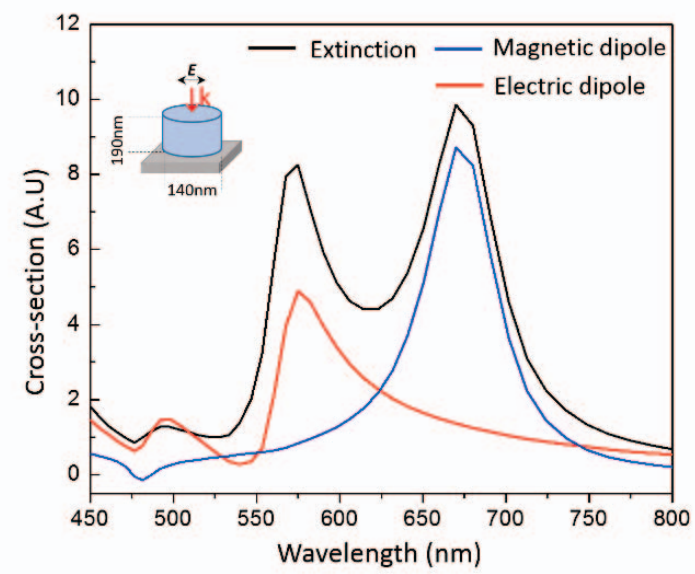

Fig. 6. Calculated extinction spectra of single silicon nanorod.

At normal incidence, the coupled dipole model yields the following expression for the reflectance [4].

$$
\begin{aligned}
& |r|^{2}=\frac{k_{0}^{2}}{4 S_{L}^{2}}\left(\left[\operatorname{Re}\left(\alpha_{e f f}^{E}\right)-\operatorname{Re}\left(\alpha_{e f f}^{M}\right)\right]^{2}+\left[\operatorname{Im}\left(\alpha_{e f f}^{E}\right)-\operatorname{Im}\left(\alpha_{e f f}^{M}\right)\right]^{2}\right) \\
& S=\sum_{(i, j) \neq(0,0)}\left[\frac{\left(1-i k_{0} r_{i j}\right)\left(3 \cos ^{2} \theta_{i j}-1\right) e^{i k_{0} r_{j}}}{r_{i j}^{3}}+\frac{k_{0}^{2} \sin ^{2} \theta_{i j} e^{i k_{0} r_{j}}}{r_{i j}}\right] \\
& \alpha_{e f f}^{E}=\frac{1}{\frac{1}{\alpha^{E}}-S} \quad \alpha_{e f f}^{M}=\frac{1}{\frac{1}{\alpha^{M}}-S}
\end{aligned}
$$

where $S_{L}$ is the area of the unit cell, $\alpha^{\mathrm{E}}$ and $\alpha^{\mathrm{M}}$ are the electric and magnetic dipole polarizabilities, respectively, $k_{0}$ is the wavevector, and $\theta_{i j}$ is the angle between the incident electric field polarization and the position vector $r_{i j}$, which is the location of the dipole with index $(i, j)$.

\section{CONCLusions}

Due to operating in the visible portion of the spectrum, we anticipate several potential applications for our silicon metasurface, including document security, LEDs, solar energy and single molecule detection.

\section{REFERENCES}

[1] A. I. Kuznetsov, A. E. Miroshnichenko. Y. H. Fu, J. Zhang and B. Luk'yanchuk "Magnetic light" Sci. Rep., 2, 492 (2012)

[2] Sheng Liu, Michael B. Sinclair, Thomas S. Mahony, Young Chul Jun, Salvatore Campione, James Ginn, Daniel A. Bender, Joel R. Wendt, Jon F. Ihlefeld, Paul G. Clem, Jeremy B. Wright, and Igal Brener "Optical magnetic mirrors without metals" Optica, 1, 250 (2014)

[3] I. Staude. A.E. Miroshnichenko, M. Decker, N.T. Fofang, S. Liu, E. Gonzales, J. Dominguez, T.S. Luk, D.N. Neshev, I. Brener, and Y. Kivshar., "Tailoring directional scattering through magnetic and electric resonance in subwavelength silicon nanodisks", ACS Nano, 7, 7824 (2013)

[4] A.B. Evlyukhin, C. Reinhardt, A. Seidel, B.S. Luk'yanchuk, and B.N. Chichkov, "Optical response features of $\mathrm{Si}-$ nanoparticle arrays,” Phys Rev B 82, 045404 (2010) 